\title{
Pathogen Profile of Patients with Sepsis in Internal Medicine Dr. Hasan Sadikin General Hospital, Bandung 2013
}

\author{
Afiq Syazwan Fauzi, ${ }^{1}$ H. Uun Sumardi, ${ }^{2}$ Nina Tristina ${ }^{3}$ \\ ${ }^{1}$ Faculty of Medicine Universitas Padjadjaran, ${ }^{2}$ Department of Internal Medicine Faculty of \\ Medicine, Universitas Padjadjaran/Dr. Hasan Sadikin General Hospital, Bandung, ${ }^{3}$ Department \\ of Clinical Pathology Faculty of Medicine Universitas Padjadjaran/Dr. Hasan Sadikin General \\ Hospital, Bandung,
}

\begin{abstract}
Background: Sepsis is a continuous disease which begins with systemic inflammatory response syndrome (SIRS), seen in association with a large number of clinical conditions. These include infectious insults that produce SIRS, such as pancreatitis, ischemia, multiple traumas and tissue injury, hemorrhagic shock, immunemediated organ injury, and the exogenous administration of such putative mediators of the inflammatory process as tumor necrosis factor and other cytokines. A frequent complication of SIRS is the development of organ system dysfunction, including such well-defined clinical conditions as acute lung injury, shock, renal failure, and multiple organ dysfunction syndrome (MODS). Hence, this study was conducted to identify the pathogen profile that often causes sepsis.

Methods: A retrospective study was performed to 152 medical records of patients diagnosed as sepsis from Internal Medicine Department Dr. Hasan Sadikin General Hospital from January 2013 to December 2013. The variables observed from the medical records were age, sex, comorbidity, main infection, culture sample, type of gram bacteria, resistant bacteria, and antibiotic susceptibility test. After data collection was completed, the data were analyzed using computer. The data were presented in percentage.

Results: Sepsis in male was higher than female. Highest comorbid was chronic kidney disease (CKD). The main infection was health care acquired pneumonia (HCAP). Highest pathogen that caused sepsis was Escherichia coli and highest multidrug-resistant organism (MDRO) was extended spectrum beta-lactamase (ESBL) Escherichia coli.
\end{abstract}

Conclusions: The most common pathogen that causes sepsis is Escherichia coli. [AMJ.2016;3(2):200-5]

Keywords: Chronic kidney disease, Escherichia coli, sepsis

\section{Introduction}

The mortality caused by sepsis, particularly related to organ dysfunction, remains a priority to clinicians worldwide and deserves greater public health attention. ${ }^{1}$ Systemic Inflammatory Response Syndrome (SIRS) is a syndrome occurring in a patient having systemic response symptoms such as fever or hypothermia, leukocytosis or leukopenia, tachypnea and tachycardia. ${ }^{1}$

Patients with sepsis are classically considered to be patients who have a high risk of morbid complications and death. This is in a large part owing to the organ dysfunction caused by sepsis, and the associated complications of organ dysfunction. ${ }^{3}$ Septic patients tend to be high resource consumers in hospitals and ICUs, and their presence affects the outcomes of those ICUs overall.4 Estimation from around the world that consistently report cases of sepsis costs from US $\$ 25,000$ to $\$ 50,000$ per episode. ${ }^{4}$ In some instances, bacteria can be resistant to multiple drugs; these strains are considered multi-drug resistant (MDR). ${ }^{5}$

Every year, thousands of patients are admitted to Dr. Hasan Sadikin General Hospital. High percentages of them are admitted for episodes such as trauma, respiratory infections or gastrointestinal infections. Blood culture examination remains as the 'Gold Standard' for the detection of bacteria and fungi. ${ }^{2}$ Blood cultures are important in defining local spectra of pathogens and resistance in severe infections. This study was conducted to identify bacteria profile of patients with sepsis at Dr. Hasan Sadikin General Hospital.

Correspondence: Afiq Syazwan Fauzi, Faculty of Medicine, Universitas Padjadjaran, Jalan Raya Bandung-Sumedang Km.21, Jatinangor, Sumedang, Indonesia, Phone: +628998000352 Email: afiqsyazz@gmail.com 


\section{Methods}

A retrospective study was performed to medical records of patients diagnosed as sepsis from Internal Medicine Department Dr. Hasan Sadikin General Hospital from January
2013 to December 2013. The variables observed from the medical records were age, sex, comorbidity, main infection, culture sample, type of gram bacteria, resistant bacteria, and antibiotic susceptibility test. Descriptive analysis for pathogen pattern and resistant MDRO pattern were compiled.

Table 1 Distribution of Patients Accordance to Sex, Comorbidity, Diagnosis of Infection, Pathogen Involved and MDRO

\begin{tabular}{|c|c|c|c|}
\hline \multicolumn{2}{|l|}{ Factors } & \multirow{2}{*}{$\frac{\text { Frequency }(\mathbf{n}=\mathbf{1 0 0})}{84}$} & \multirow{2}{*}{$\frac{\text { Percentage (\%) }}{55.3}$} \\
\hline Sex & Male & & \\
\hline & Female & 68 & 44.7 \\
\hline \multirow[t]{8}{*}{ Comorbidity } & Burn Injury & 6 & 3.9 \\
\hline & CAD & 5 & 3.3 \\
\hline & CKD & 30 & 19.7 \\
\hline & DM & 17 & 11.2 \\
\hline & GBS & 5 & 3.3 \\
\hline & Peritonitis & 6 & 3.9 \\
\hline & Pleural Effusion & 5 & 3.3 \\
\hline & TB & 7 & 4.6 \\
\hline \multirow[t]{8}{*}{ Main Infection } & HCAP & 34 & 22.4 \\
\hline & HAP & 15 & 9.9 \\
\hline & CAP & 4 & 2.6 \\
\hline & UTI & 33 & 21.7 \\
\hline & Cellulitis & 30 & 19.7 \\
\hline & BSI & 2 & 1.3 \\
\hline & Perforation & 28 & 18.4 \\
\hline & Meningitis & 6 & 3.9 \\
\hline \multirow[t]{6}{*}{ Pathogen } & Staphylococcus epidermidis & 7 & 4.6 \\
\hline & Staphylococcus emolyticus & 12 & 7.9 \\
\hline & Staphylococcus hominis & 8 & 5.3 \\
\hline & Klebsiella pneumoniae & 25 & 16.4 \\
\hline & Pseudomonas aeruginosa & 15 & 9.9 \\
\hline & Escherichia coli & 32 & 21.1 \\
\hline \multirow[t]{7}{*}{ MDRO } & MDR Pseudomonas & 16 & 10.5 \\
\hline & ESBL Escherichia & 32 & 21.1 \\
\hline & ESBL Klebsiella & 17 & 11.2 \\
\hline & Non ESBL Escherichia & 6 & 3.9 \\
\hline & Non ESBL Klebsiella & 8 & 5.3 \\
\hline & MDR Staphylococcus & 22 & 14.5 \\
\hline & Non MDR Staphylococcus & 9 & 5.9 \\
\hline
\end{tabular}

Note: $\mathrm{CAD}=$ coronary artery disease, $\mathrm{CKD}=$ chronic kidney disease, $\mathrm{DM}=$ diabetes mellitus, $\mathrm{GBS}=$ Guillain-Barre syndrome, $\mathrm{TB}=$ tuberculosis, $\mathrm{HCAP}=$ health care acquired pneumonia, $\mathrm{HAP}=$ hospital acquired pneumonia, $\mathrm{CAP}=\mathrm{community}$ acquired pneumonia, UTI=urinary tract infection, $\mathrm{BSI}=$ blood stream infection, $\mathrm{MDRO}=$ multi drug resistant organism, $\mathrm{MDR}=$ multi drug resistant, ESBL=extended spectrum beta-lactamase 
Table 2 Drug Resistance Profile against 3rd Generation Cephalosporin Antibiotics

\begin{tabular}{lcccc}
\hline \multicolumn{1}{c}{ MDRO } & Resistance & Sensitive & Not tested & Total \\
\hline MDR Pseudomonas & 16 & 0 & 0 & 16 \\
ESBL Escherichia & 31 & 1 & 0 & 32 \\
ESBL Klebsiella & 17 & 0 & 0 & 17 \\
Non-ESBL Escherichia & 1 & 5 & 0 & 6 \\
Non-ESBL Klebsiella & 0 & 8 & 0 & 8 \\
MDR Staphylococcus & 22 & 0 & 0 & 22 \\
Non MDR Staphylococcus & 0 & 9 & 0 & 9 \\
\hline
\end{tabular}

Note: MDRO=multidrug-resistant organism, MDR=multidrug-resistant, ESBL=extended spectrum beta-lactamase

After data collection was completed, the data were analyzed using computer. The data were presented in percentage, with permission from the Health Research Ethics Committee Faculty of Medicine Universitas Padjadjaran to perform the study.

\section{Results}

From the 334 medical records, only 152 of them consisted of complete variables requested. The average of patient age was 43.57 (13.055). The minimum age was 15 years old and the maximum age was 60 years old.

Distribution of sex, comorbidity, main infection, pathogen, and MDRO are presented in Table 1.

Based on Table 1, the occurrence of sepsis was higher in male (55.3\%) compared to female (44.7\%). The highest comorbidity in this study was chronic kidney disease (19.7\%), followed by diabetes mellitus (11.2\%). The highest main infection was health care acquired pneumonia (HCAP) $(22.4 \%)$, followed by $21.7 \%$ urinary tract infection (UTI) and $19.7 \%$ cellulite. The most common pathogen that caused sepsis was Escherichia coli (21.1\%) and Klebsiella pneumonia (16.4\%). Extended spectrum betalactamases (ESBL) Escherichia coli was found to be the highest multidrug-resistant organism (MDRO) (21.1\%). The second most common MDRO was MDR Staphylococcus (14.5\%), followed by ESBL Klebsiella (11.2\%).

The resistant MDRO patterns were presented in Table 2, Table 3, Table 4 and Table 5.

The MDR Pseudomonas, ESBL Escherichia coli, ESBL Klebsiella pneumonia and MDR Staphylococcus were resistant to third generation cephalosporin such as Cefotaxime and Ceftriaxone.

The MDR Pseudomonas aeruginosa was sensitive to fourth generation cephalosporin like cefepime and ceftazidime only four MDR Pseudomonas aeruginosa. All MDRO pathogens were more susceptible to Amikacin antibiotics as presented in Table 4.

All MDRO pathogens were more susceptible to Meropenem antibiotics as presented in Table 5 .

\section{Discussions}

The average age of patients from this study

Table 3 Drug Resistance Profile against 4th Generation Cephalosporin Antibiotics

\begin{tabular}{lcccc}
\hline \multicolumn{1}{c}{ MDRO } & Resistance & Sensitive & Not tested & Total \\
\hline MDR Pseudomonas & 12 & 4 & 0 & 16 \\
ESBL Escherichia & 32 & 0 & 0 & 32 \\
ESBL Klebsiella & 17 & 0 & 0 & 17 \\
Non-ESBL Escherichia & 1 & 5 & 0 & 6 \\
Non-ESBL Klebsiella & 0 & 8 & 0 & 8 \\
MDR Staphylococcus & 22 & 0 & 0 & 22 \\
Non-MDR Staphylococcus & 0 & 9 & 0 & 9 \\
\hline
\end{tabular}

Note: MDRO=multidrug-resistant organism, MDR=multidrug-resistant, ESBL=extended spectrum beta-lactamase 
Table 4 Drug Resistance Profile against Amikacin Antibiotics

\begin{tabular}{lcccc}
\hline \multicolumn{1}{c}{ MDRO } & Resistance & Sensitive & Not tested & Total \\
\hline MDR Pseudomonas & 2 & 14 & 0 & 16 \\
ESBL Escherichia & 0 & 32 & 0 & 32 \\
ESBL Klebsiella & 2 & 15 & 0 & 17 \\
Non-ESBL Escherichia & 0 & 6 & 0 & 6 \\
Non-ESBL Klebsiella & 0 & 8 & 0 & 8 \\
MDR Staphylococcus & 0 & 0 & 22 & 22 \\
Non-MDR Staphylococcus & 0 & 9 & 0 & 9 \\
\hline Note: MDR0=multidrug-resistant organism, MDR=multidrug-resistant, ESBL=extended spectrum beta-lactamase
\end{tabular}

was 43.57 years old with minimum age was 15 years old and maximum age was 60 years old. The incidence of sepsis increases disproportionately in older adults and more than half of severe sepsis cases occur in adults aged over 50 years old. ${ }^{6}$ On top of that, more than half of patients who develop severe sepsis also have at least one chronic health condition.

Male appears to be at higher risk of developing sepsis compared to female. ${ }^{7}$ The fact that the occurrence is higher in male compared to female may be explained by a combination of differences in chronic disease burden, particularly subclinical disease, social and environmental factors, and genetic predisposing causing difference in the host immune response towards infection that likely contributes to the observed difference. For example, a few healthy female volunteers showed a more pronounced pro-inflammatory response after endotoxin infusion compared to healthy men. ${ }^{8}$ On top of that, men usually tend to be treated by undergoing more invasive procedures whereas women frequently tend to not undergo invasive procedures. ${ }^{9}$ The role of estrogens and androgens in female and male respectively may account for the sex differences in sepsis outcomes where low levels of estrogen and high levels of androgens are more prone to infections. ${ }^{9}$

The most common comorbidity that was found in these septic patients was CKD $(19.7 \%)$. However, in previous research, it showed that trauma or having history of previous surgery is the highest comorbid that was found in septic patients. ${ }^{10}$ This may be explained by the fact that after a traumatic injury or a previous surgery, the body produces a flood of white blood cells that can secrete a protein known as HMGB1. This protein contributes to septic inflammation which can be life-threatening. ${ }^{10}$

For the main infection in this research, HCAP was the highest occurrence (22.4\%). However, the highest main infection from previous research is CAP. This may be explained by the observation that patients with HCAP usually are from low socioeconomical level. These individuals are usually more susceptible to infections. Patients with HCAP usually have low immune system level due to incomplete vaccination or have not had any vaccination before. $^{10}$

Gram-negative organism was shown to be the largest pathogen that causes

Table 5 Drug Resistance Profile against Meropenem Antibiotics

\begin{tabular}{lcccc}
\hline \multicolumn{1}{c}{ MDRO } & Resistance & Sensitive & Not tested & Total \\
\hline MDR Pseudomonas & 1 & 15 & 0 & 16 \\
ESBL Escherichia & 0 & 32 & 0 & 32 \\
ESBL Klebsiella & 2 & 15 & 0 & 17 \\
Non-ESBL Escherichia & 0 & 6 & 0 & 6 \\
Non ESBL Klebsiella & 0 & 8 & 0 & 8 \\
MDR Staphylococcus & 20 & 2 & 0 & 22 \\
Non-MDR Staphylococcus & 0 & 9 & 0 & 9 \\
\hline
\end{tabular}

Note: MDRO=multi drug resistant organism, MDR=multi drug resistant, ESBL=extended spectrum beta-lactamase 
sepsis. ${ }^{11}$ Escherichia coli (21.1\%) was the highest gram-negative organism followed by Klebsiella pneumonia $(16.4 \%)$ and Pseudomonas aeruginosa (9.9\%). For grampositive organism, the highest pathogen was Staphylococcus hemolyticus $(7.9 \%)$ followed by Staphylococcus hominis $(5.3 \%)$ and Staphylococcus epidermidis (4.6\%). This is because, the gram-positive bacteria cell wall is composed of peptidoglycans and has two layers only while gram-negative bacteria cell wall is more complex as it has an outer membrane, a space and a layer of peptidoglycans. On top of that, the gram-negative bacteria cell wall also contains pore proteins which it can pump substance back out of the bacterium if it is harmful towards it. ${ }^{5}$ Hence, that is why gramnegative bacteria are more likely to cause infection towards a person compared to grampositive bacteria. ${ }^{5.12}$

It was discovered that ESBL Escherichia coli isthe highest pathogen that causes MDR. This may be explained by which acquisition of Escherichia coli's genes that produces ESBLs, an enzyme that breaks down antibiotic and, hence, prevent them from working. ${ }^{5}$ Escherichia coli are also able to change its cell wall structure until it prevents antibiotics from entering or limiting their activity against the cell wall, and the pore protein on its cell wall surface can affect most classes of drugs; these mechanisms are also seen in Enterobacter species. $^{5}$

Third and fourth generation cephalosporin antibiotics are resistant against ESBL Escherichia coli while Amikacin and Meropenem antibiotics are sensitive against ESBLEscherichia coli. Previous research proved that Carbapenems are the drugs of choice for many infections caused by gam-negative and gram-positive bacteria and are found to be the most effective antibiotics which are the same in this research. ${ }^{13}$ However, previous research showed that low resistance to cephalosporins was seen. This may be explained due to plasmids harboring several resistance genes which are transferred from one bacterium to another and have linked such resistance pattern to the presence of integrons. ${ }^{13}$

Amikacin and Meropenem were sensitive in this study. Amikacin binds to components of bacteria that produce important bacterial proteins, blocking protein synthesis which eventually leads to stopping further bacterial growth. ${ }^{14}$ Amikacin is used to treat infections caused by bacteria that are resistant to gentamicin and tobramycin. Amikacin treats infections caused by gram-negative bacteria such as Pseudomonas species, Escherichia coli, Providencia species, Indolepositive and indole-negative Proteus species, Klebsiella-Enterobacter-Serratia species, and Acinetobacter. Amikacin is also used in certain staphylococcal infections as well. ${ }^{13,14}$

Meropenem is a broad-spectrum antibacterial agent of the carbapenem family, indicated as empirical therapy prior to the identification of causative organisms, or for disease caused by single or multiple susceptible bacteria in both adults and children with a broad range of serious infections. ${ }^{14}$ Meropenem showed greater efficacy than ceftazidime or piperacillin/tazobactam in febrile neutropenia, and greater efficacy than ceftazidime plus amikacin or tobramycin in patients with nosocomial pneumonia. Meropenem is well-tolerated and has the advantage of being suitable for administration as an intravenous bolus or infusion. Its low propensity for inducing seizures means that it is suitable for treating bacterial meningitis and is the only carbapenem approved in this indication. Thus, meropenem continues to be an important option for the empirical treatment of serious bacterial infections in hospitalized patients. ${ }^{15}$

There were some limitations of the study. Not all of the medical records consisted of the variables requested, only $30 \%$ of the medical records met the requirement. Other limitation was the outcomes of the treatment were not recorded. Hence, without these data, it is hard to know the successful rate of treatment towards the patients. As a recommendation, end point of septic patient's treatment should be recorded so that we can know the successful rate of antibiotic treatment. Moreover, medical records should be written completely.

It can be concluded that the most common pathogens that cause sepsis are Escherichia coli, Klebsiella pneumonia, Pseudomonas aeruginosa and Staphylococcus species. Theses pathogens are also resistant against antibiotics due to their special characteristics.

\section{References}

1. Martin GS. Sepsis, severe sepsis and septic shock: changes in incidence, pathogens and outcomes. Expert Rev Anti Infect Ther. 2012;10(6):701-6.

2. Lin EC, Boehm KM. Positive predictive value of blood cultures utilized by community emergency physicians. Int Scholarly Res Notices Infect Dis. 2013;2013(135607): $1-5$. 
3. Degoricija V, Sharma M, Legac A, Gradiser M, Sefer S, Vucicevic Z. Survival analysis of 314 episodes of sepsis in medical intensive care unit in university hospital: impact of intensive care unit performance and antimicrobial therapy. Croat Med J. 2006;47(3):385-97.

4. Moerer O, Plock E, Mgbor U, Schmid A, Schneider $\mathrm{H}$, WischnewskyMB, et al. A German national prevalence study on the cost of intensive care: an evaluation from 51 intensive care units. Crit Care. 2007;11(3):R69.

5. Tam VH, Rogers CA, Chang KT, Weston JS, Caeiro JP, Garey KW. Impact of multidrug-resistant Pseudomonas aeruginosa bacteremia on patient outcomes. Antimicrob Agents Chemother. 2010;54(9):3717-22.

6. Vincent JL, Rello J, Marshall J, Silva E, Anzueto A, Martin CD, et al. International study of the prevalence and outcomes of infection in intensive care units. JAMA. 2009;302(21):2323-9.

7. Sakr Y, Elia C, Mascia L, Barberis B, Cardellino S, Livigni S, et al. The influence of gender on the epidemiology of and outcome from severe sepsis. Crit Care. 2013;17(2):R50.

8. van Eijk LT, Dorresteijn MJ, Smits P, van der Hoeven JG, Netea MG, Pickkers P. Gender differences in the innate immune response and vascular reactivity following the administration of endotoxin to human volunteers. Crit Care Med. 2007;35(6):1464-9.

9. Eachempati SR, Hydo L, Shou J, Barie PS. Sex differences in creation of do-notresuscitate orders for critically ill elderly patients following emergency surgery. J Trauma. 2006;60(1):193-8.

10. Ortiz G, Duenas C, Rodriguez F, Barrera L, de La Rosa G, Dennis R, et al. Epidemiology of sepsis in Colombian intensive care units. Biomedica. 2014;34(1):40-7.

11. Vincent JL, Sakr Y, Sprung CL, Ranieri VM, Reinhart K, Gerlach H, et al. Sepsis in European intensive care units: results of the SOAP study. Crit Care Med. 2006;34(2):344-53.

12. Phua J, Ngerng W, See K, Tay C, Kiong T, Lim H, et al. Characteristics and outcomes of culture-negative versus culture-positive severe sepsis. Crit Care. 2013;17(5):R202.

13. Manjula NG, Math GC, Nagshetty K, Patil SA, Gaddad SM, Shivannavar CT. Antibiotic susceptibility pattern of ESBLproducing Klebsiellapneumoniaeisolated from urine samples of pregnant women in Karnataka. J Clin Diagn Res. 2014;8(10):DC08-11.

14. Brooks G, Carrol K, editors. Antimicrobial chemotherapy. 25th ed. New York: McGraw-Hill; 2010.

15. Baldwin CM, Lyseng-Williamson KA, Keam SJ. Meropenem: a review of its use in the treatmen of serious bacterial infections. Drugs. 2008;68(6):803-38. 